ISSN electrónico: 2172-9077

https://doi.org/10.14201/fjc201715199217

\title{
¿Digo LO QUE SIENTO Y SIENTO LO QUE DigO? UNA APROXIMACIÓN TRANSCULTURAL AL USO DE LOS EMOTICONOS Y EMOJIS EN LOS MENSAJES EN CMC
}

\section{Do I Mean What I Say and Say What I mean? A Cross Cultural Approach to the Use of Emoticons \& Emojis in CMC Messages}

\author{
Dra. Lifen CHENG \\ Profesora titular de la Universidad de Salamanca (España) \\ E-mail:1 1fcheng@,usal.es
}

Fecha de recepción del artículo: 01/06/2017

Fecha de aceptación definitiva: 23/10/2017

\begin{abstract}
RESUMEN
El uso de los emojis y emoticonos entre los usuarios de mensajería online es un fenómeno global y generalizado. El presente estudio analiza las preferencias entre los usuarios de unos emojis y emoticonos sobre otros con el fin de adecuar mejor la expresión de sus mensajes con la emoción que contienen. Lo hacen así para intensificar o difuminar la rigidez textual. También, para manifestar su propia empatía durante las interacciones del proceso comunicativo. Este trabajo analiza el uso de estos símbolos y elementos gráficos en el intercambio de mensajes online por medios sociales; a través de ellos los usuarios, según su diferente procedencia cultural, van a trasmitir aquellos estados emocionales que más se sientan afectados. Para ello, se han llevado a cabo estudios empíricos basados en un diseño metodológico que consta de dos fases de investigación: Un estudio piloto para la validación del instrumento investigador y un segundo estudio basado en encuesta. Los resultados observados indican que la procedencia cultural podría influir, hasta cierto punto, en el grado de expresividad de su gente a la hora de elegir entre 1) el uso de texto verbal único, 2) los elementos de signo en el teclado, 3) la inclusión de un emoticono o, 4) un emoji facial expresivo, cuando utilizan la mensajería online de medios y redes sociales para comunicar sus sentimientos o emociones.
\end{abstract}

Palabras clave: CMC; emojis; emoticonos; SNS; TIC; variabilidad cultural.

\begin{abstract}
The use of emoticons and emojis among online messaging users has achieved a globalized level. This article aims at examining how certain emojis and emoticons are chosen by message senders to regular the intensity of emotions in their messages. Or, by means of using them, message senders wish to blur textual rigidity and show interaction empathy during the communication process. This study analyzes the use of these symbols and graphic elements in message exchange through CMC and SNS, where users from different culture background convey their feeling. For it, two empirical studies of a methodological design were carried out: One pilot study was intended for the research instrument validation. The second was a survey study. By testing online messaging habit, this study enabled the researchers to observe whether the senders inclined to linguistic text use only, or they preferred including an additional emoticon or emoji in their message to communicate their feelings. Results of this research show that, at a certain extent, massage senders' different culture background could influence on their emotional expressivity level.
\end{abstract}

Key words: CMC; culture variability; emojis; emoticons; SNS; TICs. 


\section{INTRODUCCIÓN}

El uso de las aplicaciones de las tecnologías de información y comunicación (TIC) se ha instalado hoy en día en la vida cotidiana de muchos habitantes del planeta. En la última década, el acceso a Internet ha sido adoptado por el público general como vehículo social para sus interacciones interpersonales. CMC, siglas de Comunicación Mediada por Computador, es un término genérico que incluye todas las comunicaciones realizadas mediante redes y mantenidas por dichas tecnologías, cuyo desarrollo y expansión es además continua en todos los rincones del planeta y en todas las capas sociales.

Esencialmente, la CMC es definida como cualquier comunicación humana a través del uso de dos o más dispositivos electrónicos. Mientras que este término ha hecho siempre referencia a aquellas comunicaciones que transcurren por vía de formatos mediados por computador, también se ha aplicado a otras formas de interacción basada en el envío de mensajes textuales. Hasta hace poco, la mayoría de la investigación en CMC enfocaba los estudios sobre los efectos sociales de las diferentes tecnologías de comunicación con soporte de ordenadores (Adrianson, 2001), mientras que muchos de los más recientes se interesan por redes sociales con soporte de software social. En este sentido, se considera la CMC como un proceso donde ocurre una interacción de los datos humanos mediante uno o más sistemas de telecomunicación conectados en red y software. Estos incluyen: Email, Internet Relay Chat (IRC), instant messaging (IM), Usenet (Users Network o Red de usuarios), LISTSERVs (mailing list servers -listas de distribución que prestan un servicio de Internet basado en el correo electrónico y que reúnen un conjunto de direcciones electrónicas usadas para enviar mensajes o anuncios a los miembros de la lista-) así como los SMS, acrónimo de Short Message Service en inglés, sistema de mensajes de texto para teléfonos móviles, además de SNS (Social Networking Service) o el servicio de red social en inglés.

La CMC se puede desarrollar en modo sincrónico y en modo asincrónico. En el primero, todos los participantes concurren en línea simultáneamente. En el segundo, existen restricciones en el tiempo entre el envío de mensajes y la recepción de respuestas, como el caso de los emails. Las características clave de CMC posibilitan registrar y recuperar la conversación, la comunicación formal o/y el anonimato de identidad del usuario, dependiendo del tipo de software - por ejemplo, IM -instant messaging (mensajería instantánea). Sin embargo, puede que las emociones detrás de una mera aseveración del usuario resulten difíciles de interpretarse debido a la ausencia de la comunicación de cara a cara. La falta de contacto visual puede generar malentendidos como efectos negativos no pretendidos, por tanto, los emoticonos proporcionan una herramienta preciosa para llenar este vacío.

Este aspecto parece haberse superado parcialmente con la posibilidad de incluir los emoticonos y emojis en los mensajes digitales, aunque no está muy clara cuál podría ser la consecuencia de su uso habitual: ¿Están cambiando los usuarios su forma de pensar? ¿Se han modificado nuestros cerebros durante los últimos años con la aparición de los emoticonos y emojis? ¿Están cambiando los patrones del habla? Tanto los emoticonos como los emojis son reconocidos y procesados por el cerebro como información no verbal, lo que significa que los «leemos» como una parte de la comunicación emocional. Y una cuestión más: ¿Su práctica difiere en las diversas culturas?

Esta última cuestión centra el interés principal del presente estudio, enfocado en analizar si existe preferencia diferenciada en el uso de los emoticonos, emojis o texto verbal único entre los comunicantes de diferentes culturas a la hora de expresar sus emociones o empatía. 


\section{REVISIÓN DE LITERATURA}

\section{Origen de los Emoticonos y Emojis}

Aunque se usan indistintamente y, a menudo, como sinónimos, lo cierto es que son cosas diferentes. El término «emoticono» es un neologismo que proviene de emoción e icono y que se refiere a emoción e imagen. Se utilizan frecuentemente en CMC donde son eliminados los factores característicos propios de la comunicación cara-a-cara tales como el ritmo de la dicción, gestos, espacio de distancia corporal, feedback inmediato del interlocutor, etc. (Dresner y Herring, 2010). La teoría de la presencia social y teorías de la comunicación postulan que para que la comunicación ocurra eficazmente es necesario que los participantes cuenten con una vía para el feedback inmediato o utilicen el lenguaje natural (Ekman, 1993). En general, CMC carece de canales disponibles y claves para una comunicación efectiva. Esto se debe a la ausencia del contexto social aplicado como una referencia teórica. Algunos investigadores argumentan que los usuarios de CMC han desarrollado nuevos signos no-verbales, como las expresiones para-lingüísticas que se denominan «emotex» o «emoticonos» para superar tales limitaciones y, de hecho, son utilizados por muchos usuarios o internautas en las comunicaciones en Internet. Ferris (1996, p. 34) define emotex como señales no-verbales para enfatizar la emoción y sugiere que emotex consta de sustitutos léxicos para los signos no-verbales. En este sentido, como el término indica, los emoticonos suponen unos arreglos visuales con las características de un texto que contienen símbolos para expresar los diferentes tipos de emoción. Se atribuye la invención a Scott Fahlman, de Universidad Carnegie Mellon, EE. UU., quien al representar una cara sonriente con unos signos del teclado en el ordenador fue el primero en usar uno de estos símbolos hace tres décadas (Ptaszynski y Arak, 2010). Estos autores cuentan que Fahlman creó el símbolo smiley compuesto de tres signos :-) del teclado de su ordenador, y otro símbolo, frowny, con estos signos :-( . Posteriormente, agregó otro signo, “) ”, al simbolo frowny, como énfasis dando como resultado el angry s:-( para expresar el sentimiento de enojo.

En su origen, estas combinaciones de signos del teclado fueron utilizadas para identificar las bromas en un foro de debate científico de la computación. Posteriormente se adaptó su uso para economizar las interacciones mediadas por ordenador. Como explica el propio Fahlman en su página web (https://www.cs.cmu.edu/ sef/sefSmiley.htm), la idea surgió en 1982 como respuesta a los problemas de interpretación de los mensajes que se intercambiaban en los tableros o paneles electrónicos usados para discutir ciertos temas online entre profesores y estudiantes, quienes plantearon que sería una buena idea explicitar qué mensajes no deberían tomarse en serio, ya que al estar en un contexto online, asentado en textos, no se contaba con los datos adicionales que aportan el lenguaje corporal o el tono de voz como sí sucede en una conversación, donde esos matices corporales o auditivos pueden informar sobre la intención o sobre el tono del mensaje. Por eso, a Fahlman se le ocurrió que la secuencia de estos caracteres :-) podía ser una solución elegante ya que, además, todos los ordenadores de entonces, basados en el código ASCII, estaban equipados de estos signos. Los valoró también como modo complementario de proteger los sentimientos susceptibles de las personas y expresar, mientras, los diferentes estados de ánimo (Ptaszynski y Arak, 2010). Desde entonces, estos símbolos se han hecho populares entre miles de personas que los usan diariamente, junto con otros emoticonos creados posteriormente, como formas de expresar su sentido de humor, su acuerdo y desacuerdo o su estado de ánimo, etc.

Los «emojis», posteriormente, han supuesto un avance creativo extraordinario. Se trata de iconos unitarios y cerrados que reflejan de modo gráfico un abanico amplio de emociones, visualmente interpretadas. Sirven para expresar los estados emotivos de cada texto con base estrictamente en los signos, igual que el uso del lenguaje no-verbal se basa en la comunicación cara-a-cara. Ellos también ayudan a 
acentuar o resaltar el tono o significado durante el proceso de redactar un mensaje (Crystle, 2001). Además de hacer más visible la comunicación de un estado de ánimo (Constantin y Kalyanaraman, 2002), los emojis cumplen la función de aclarar mensajes textuales a través de las pantallas, como en la comunicación cara-a-cara lo hace el lenguaje no-verbal (Walther y D’Addario, 2001).

Los emojis han evolucionado de este modo hasta convertirse en un nuevo lenguaje. Sus diseños diversos proporcionan nuevos códigos que estimulan el aumento de su uso entre los usuarios de CMC. Los diseñadores de emojis crean continuamente personajes nuevos y exclusivos para incrementar la carta de sentimientos trasmisibles, con mayor detalle y en pro de un entendimiento más eficaz. Surgen a diario nuevos modelos de emojis en los sistemas de mensajería de Twitter, Facebook y móviles. Se pueden encontrar muchas novedades que amplían las posibilidades de compartir emojis en el chat del Facebook y los mensajes en la página personal.

Aunque los emoticonos y los novedosos emojis, de creación posterior, son populares en comunicación, no siempre son compatibles entre todos los dispositivos y, en algunos, se plasman de forma diferente. Por esta razón existen distintos emojis, en función del área cultural de cobertura de ese soporte móvil y de sus posibles usuarios.

\section{Estudios Antecedentes sobre el Uso de Emoticonos y Emojis en CMC}

Estudios anteriores sostenían que la CMC carece de indicios de interacción no-verbal y, como consecuencia, los usuarios de Internet han adoptado el uso de los emoticonos para facilitar sus comunicaciones Se cree que vendrían a sustituir a los indicios perdidos en los discursos escritos por ordenador. La literatura revisada indica que los emoticonos sirven como proveedores socio-emocionales en el contexto de $\mathrm{CMC}$ donde se ha mostrado que el uso de emoticonos y emojis ha evolucionado hasta convertirse en una parte recurrente de casi todas las formas de interacciones mediadas por ordenador (Lo, 2008). Diferentes investigadores los han estudiado desde diferentes perspectivas. Por ejemplo, el enfoque de la aproximación psicológica tiende a centrarse en la emoción y el papel desempañado por los emoticonos/emojis para la comprensión de los mensajes. Los lingüistas han analizado los emoticonos con referencia al habla, como el parámetro de la escritura, la explicación semántica y sintáctica, la clasificación funcional y los aspectos visuales. Más que ser meramente para-lenguaje del Internet, los grafemas de los emoticonos han sido comparados con los morfemas vocabularios con distinta función significativa (Pierozak en Amaghlobeli, 2012). Esto significaría que los emoticonos pueden convertirse en unidades como los morfemas, en los que podría haber una derivación, inflexión o abreviación, pero no serían unidades aisladas. Incluso, una investigación pedagógica ha propuesto la elaboración de una lengua universal basada en emoticonos y su posible introducción en el sistema educativo (Jibril y Abdullah, 2013).

En otra investigación, Tossell et al. (2012) centraron su estudio para comprender cómo los emoticonos eran empleados en los mensajes de texto. Analizaron, sobre todo, la diferencia de la variedad de los emoticonos y la frecuencia de su uso entre los hombres y mujeres que envían mensajes por redes sociales. Las diferencias encontradas indican que las mujeres envían mayor número de mensajes con emoticonos, en cambio, los hombres utilizan una gama más diversa de estos elementos. Otra evidencia muestra que los hombres los utilizan para el humor y mostrar emociones mientras las mujeres los utilizaban para sarcasmo (Lee, 2003). Por otro lado, Huffaker y Calvert (2006) analizaron los contenidos de los blogs y encontraron que los blogs masculinos tenían un mayor número de emoticonos que en los femeninos. Otros estudios señalan que las mujeres tienden a utilizar más el estilo comunicativo no-verbal en los encuentros en F2F (Friend to Friend). Derks et al. (2007) se han interesado en comprender si esto ocurre también en CMC desarrollando estudios enfocados sobre las diferencias 
entre géneros que han dado resultados mixtos. En el Internet, los hombres no utilizaban muchos emoticonos sobre los reportajes deportivos donde la mayoría de los espectadores son también hombres. No obstante, cuando los hombres se integran en grupos de géneros mixtos, es cuando utilizan los emoticonos con mayor frecuencia. Los autores sugieren que tanto hombres como mujeres buscan aclarar los estados emocionales mediante la lectura del contenido. Además, aunque las frecuencias de los emoticonos encontrados en los foros mixtos resultaron más o menos equivalentes, ambos géneros los utilizaban de forma diferente. Este hallazgo parece indicar que en todos los métodos utilizados para analizar los procesos de CMC, no hay diferencias sistemáticas entre los géneros porque los emoticonos son utilizados de manera diferente según tareas, contextos y medios; lo mismo sucede con las expresiones faciales y con otras comunicaciones no-verbales, que son recurrentes para comunicar los indicios sociales, la emoción y para aclarar el significado de los mensajes (Derks, et al., 2007).

Un hecho importante observado por los investigadores es la falta de información contextual en los procesos de CMC que, como medio en sí, resulta perturbador para la comprensión del contenido y del carácter de los mensajes. Esta carencia de contexto ha sido considerada por los remitentes como culpable de inducir a la percepción de los mensajes electrónicos como antipáticos y ofensivos (Jenson, 2005; Derks, et al., 2007) demostrando que los emoticonos pueden suplir esta información para mejorar el entendimiento en la CMC. Los emoticonos se definen como representaciones gráficas de expresiones faciales que están incrustados en los mensajes electrónicos. Derks et al. (2007) también han encontrado que los emoticonos son usados más frecuentemente en comunicaciones sincrónicas. Esto indica que la inmediatez de su uso puede fomentar una mejor relación comunicativa. En otros estudios empíricos se comprobó que la visualización online de textos electrónicos sin emoticonos condujo a la interpretación incorrecta del carácter del mensaje y la actitud de los remitentes (Lo, 2008). La inclusión de los emoticonos ayudaba a los lectores a comprender mejor el nivel y la dirección del contexto emocional que rodeaba el mensaje transmitido por Internet. En la mensajería instantánea, los emoticonos positivos eran usados con mayor frecuencia que los negativos y su uso intensifica la valencia del mensaje. En dicho estudio, los emoticonos fueron identificados principalmente como una forma de expresar la emoción, fortalecer los mensajes y mostrar el humor o sarcasmo.

Rezabek y Cochenour (1998) analizaron los correos electrónicos a través del programa LISTSERV para averiguar el contenido de los emoticonos y la frecuencia de su uso. Estos investigadores observaron que entre 1-25\% de los correos recopilados utilizaron emoticonos y que el LISTSERV con el mayor número de mensajes analizados solo contenía un 6\% de estos. Posteriormente, Ling (2005) examinó 882 mensajes mediante entrevistas telefónicas para recoger datos y encontró que solo un 6\% de ellos contenían emoticonos. Argumentaban los autores que existían muchos factores que podrían haber influido en la varianza tan distante entre unos correos y otros, como la fuerza del vínculo social, género, edad y geografía y que, sin embargo, no fueron evaluados. Debido a la brevedad de las comunicaciones de SMS (Short Message Service) y por el hecho de que se utiliza tanto sincrónica como asincrónicamente (Kasesniemi y Rautianen, 2002), es posible que el empleo de los emoticonos tenga diferentes patrones de uso o amplificación de importancia.

Otra investigación internacional más reciente, basada en una encuesta desarrollada sobre una muestra de sujetos chinos, ha concluido que un $88 \%$ de los encuestados utilizaba emoticonos (Qiao, 2010). Estos usuarios preferían SMS más que otros medios y comunicaciones de la plataforma de F2F para expresar emociones a los demás en sus redes sociales. Los hallazgos también muestran que la mayoría de estos usuarios utilizaban emoticonos para el humor y como sustituto a la comunicación de cara a cara no verbal. 


\section{Estilo Cultural en Comunicación y el Uso de Emoticonos}

La gente aprende a comunicar los pensamientos, sentimientos y valores de su cultura compartida (Gudykunst, 1997; Gudykunst, et al., 2005). El continuo individualismo-colectivismo (Gudykunst, et al., 1996; Hall, 1976; Hofstede y Bond, 1984; Hofstede, et al., 2010), como un indicador cultural en particular, ha sido considerado por los investigadores transculturales el factor más importante en la explicación sobre la manera en que la cultura puede influir en los estilos comunicativos de los individuos. Estos incluyen el uso del lenguaje, la auto-representación y las señales no-verbales. Se espera que la cultura moldee la comunicación verbal y no-verbal. Basándose en estudios empíricos, Gudykunst et al. (1996) sostienen que los estilos usados por los individuos para comunicar varían según las culturas de donde procedan (p. 511).

Los investigadores en comunicación transcultural e intercultural proponen que las culturas pueden clasificarse a través de las diferentes maneras de comunicarse los miembros en sus distintas comunidades culturales (Hall y Hall, 1990). Las culturas nórdicas caracterizadas de individualistas, como las de Alemania, Países Bajos, Austria, etc., donde la gente tiende a comunicarse de forma más directa, son consideradas pertenecientes a la cultura de «bajo contexto» (BC). Otras culturas de característica colectivista, como las latinoamericanas y, en particular, las asiáticas -tales como China, Corea y Japón, etc.- donde los nativos se inclinan a utilizar el estilo no-verbal como el lenguaje corporal y el silencio para comunicarse, son denominadas cultura de «alto contexto» (AC). Del mismo modo, Gudykunst et al. (1996) identifican el estilo comunicativo de «alto contexto cultural» con aquel que usa un lenguaje indirecto, ambiguo, conservador y controlado. En contraste, el estilo comunicativo de «bajo contexto cultural» tiende al uso de leguaje directo, dramático, abierto y dependiente del sentimiento. Según la definición de estas categorías, los autores mencionados coinciden en afirmar que la cultura china, japonesa, coreana y otras sociedades asiáticas se inclinan hacia la categoría de cultura de «alto contexto», mientras que países como Alemania, Suiza, Estados Unidos se consideran pertenecientes a la cultura de «bajo contexto». Los países mediterráneos tales como Francia, Italia, España se sitúan a medio camino entre AC culturas y BC culturas, según los investigadores transculturales. Las cuestiones planteadas por los teóricos transculturales sobre las diferencias en el estilo comunicativo entre culturas, suscitan nuestro interés por indagar en la posible existencia de patrones culturales en el uso preferente de los emoticonos entre personas procedentes de diferentes culturas.

Diferentes culturas pueden influir distintos procesos cognitivos y distintas prácticas en los individuos de esa comunidad cultural, puesto que la cultura influye sobre dicha vivencia. Los resultados obtenidos de los estudios antecedentes (Páez, et al., 2004; Fernández, et al., 2000) muestran que las reacciones emocionales son percibidas y referenciadas con mayor intensidad en aquellas personas pertenecientes a sociedades desarrolladas, en la que se valora más la cultura individualista con orientación femenina y se mantiene una estructura social con tendencia a la baja distancia de poder, etc. También, las diferentes culturas representan sus emociones de manera diferente: la expresión facial, las posturas y movimientos corporales, la mirada y el contacto físico juegan un papel significativo en la interacción social. Estas constituyen las formas visibles mediante las que se manifiestas las idiosincrasias culturales.

Por otro lado, Walther y D'Addario (2001, p. 344) concluyeron en su trabajo apuntando que los efectos comunicativos reales de un emoticono son mínimos dentro del contexto de los indicios lingüísticos a los que podría acompañar. Más tarde, Provine et al. (2007) hallaron que la expresión emocional estaba subordinada a la producción de frases habladas. No obstante, frente a esta visión, en otros estudios experimentales Derks et al. (2008) encontraron cierta influencia de los emoticonos sobre la percepción de las expresiones y mostraron que los lectores percibieron los mensajes con emoticonos como más positivos que los mensajes sin ellos. Concluyeron que estos podrían sustituir 
algunas de las funciones del comportamiento no-verbal, complementando y mejorando ese mensaje verbal, aunque carecen de la capacidad de contradecirlo.

\section{Expresión de los Emociones y la Reducción del Efecto Framing}

En un estudio experimental antecedente, Thompson y Foulger (1996) ya examinaban el efecto de emoticonos sobre la percepción de framing. Los resultados en dicho estudio mostraron que los emoticonos podían modifican la percepción de framing y alertar a los lectores de que podrían tomar el mensaje con menos seriedad. De este modo, su uso representa una estrategia útil para evitar los brotes de framing no-intencionado. Ellensburg (2012) confirma con su hallazgo que el uso muy frecuente de los emoticonos en el ámbito profesional puede reducir percepciones de poder, competencia, estatus y moralidad.

En suma, los emoticonos son básicamente imágenes gráficas de uso múltiple y diverso, que permiten a sus usuarios expresar sus sentimientos mediante la CMC. Sin embargo, todavía no se sabe con claridad qué aspectos culturales o demográficos podrían afectar su utilización en la comunicación, y en qué estado emocional los usuarios prefieren utilizar determinados emoticonos para expresar las emociones sentidas tales como enojo, alegría o tristeza.

\section{OBJETIVo GENERAL DEL ESTUdio}

El propósito de este trabajo es conocer y describir los usos de los emoticonos en comunicación bajo la posible influencia tanto de las emociones incrustadas en los mensajes, como del bagaje cultural de los usuarios. Para ello se ha diseñado un cuestionario para recabar información sobre si los emoticonos son efectivamente utilizados en los mensajes por los usuarios de SMS en CMC o SNS de diferentes procedencias culturales y, por otro lado, si existen patrones culturales en el uso de los emoticonos. Aparte de la consideración de la posible influencia de la variabilidad del estilo cultural comunicativo sobre el modelo de uso preferente de los emoticonos, el presente estudio centra especialmente el interés sobre el estado emocional de los usuarios en el momento de enviar mensajes online. Los análisis de la presente investigación enfocan sobre la cuestión de si las emociones sentidas por los usuarios podrían influir en la elección del tipo de emoticonos que los usuarios consideren más eficaz para transmitir sus sentimientos.

\section{Planteamiento de Preguntas de Investigación}

En base a los argumentos revisados en los estudios anteriores que parecen indicar que, independientemente de las diferencias culturales, la tendencia de la humanidad es mostrar su deseo universal de mantener el ánimo y el optimismo a pesar de las crisis y conflictos. Por consiguiente, el presente estudio formula las siguientes situaciones para analizar y comprenderlas:

Pregunta 1: ¿Los usuarios en general, independientemente de las procedencias culturales, que envían mensajes positivos por vía CMC también optan por acompañarlos con algún tipo de signo noverbal para transmitir y compartir su estado optimista?

Pregunta 2: En comparación con la comunicación de mensajes positivos, ¿̇los usuarios en general reducen la frecuencia del uso de los signos tristes no-verbales para comunicar mensajes negativos mediante CMC para evitar que se enfatice el sentimiento triste en dichos mensajes?

Siendo la ira o enojo un sentimiento instintivo del equilibrio moral que brota ante una injusticia y produce en el sujeto una sensación de energía o impulsividad, esto le lleva a actuar física o verbalmente de forma intensa e inmediata para solucionar de forma activa la situación problemática. En tal estado de enojo descrito arriba, se establece: 
Pregunta 3: ¿Puede que con mayor frecuencia los usuarios de CMC en general emitan directamente mensajes en texto verbal más que recurrir a los emoticonos con expresiones enojosas?

En su obra original, Hall (1976) argumentaba que los individuos procedentes de BC cultural veían la comunicación cara a cara como una forma de abordar problemas y solucionarlos. De manera opuesta, los sujetos de culturas de AC transmitirían los mensajes sin referirse de forma verbal al problema directamente, tienden a expresarse de forma no-verbal y dejar que fluya la armonía. Con estos argumentos, se formulan las siguientes preguntas a continuación:

Pregunta 4: ¿Cuáles son las preferencias de los sujetos chinos en comparación con los españoles en su utilización de los emoticonos para comunicar los mensajes sobre el «enojo» en CMC?

Pregunta 5: ¿Cuáles son las preferencias de los sujetos chinos en comparación con los españoles en su utilización de los emoticonos para comunicar los mensajes sobre la «alegría» en CMC?

Pregunta 6: ¿Cuáles son las preferencias de los sujetos chinos en comparación con los españoles en su utilización de los emoticonos para comunicar los mensajes sobre la «tristeza» en CMC?

\section{MÉTODOS}

\section{E1 Pretest}

Con el fin de comprobar el acuerdo en la identificación de los significados transportados por los emoticonos de uso más generalizado online, estos fueron sometidos a un chequeo previo para la comprobación inequívoca del mensaje emocional que cada emoticono podría emitir para los usuarios de CMC. Para el presente estudio, se utilizaron los nueve emoticonos más conocidos y más usados popularmente en CMC como instrumento para examinar la preferencia de los usuarios al expresar sus emociones en los mensajes enviados por Internet o redes sociales.

\section{- Validación del Instrumento}

Los emoticonos usados para el presente estudio fueron, en primer lugar, los tres compuestos de signos ASCII mediante teclado que representan la alegría :-), la tristeza :-( y el enojo ::-( ; segundo, las caras conocidas como smiley $\ddot{\cup}$, frowny ${ }^{\prime}$ y angry $*$, por el último, los emojis con elementos gráficos faciales expresivos alegría ${ }^{\circ}$, tristeza ${ }^{\circ}$ y enojo $\odot$.

Tabla 1. Resultados del pretest de identificación de instrumentos $(\mathrm{N}=40)$

\begin{tabular}{|c|c|c|c|c|}
\hline \multirow[b]{2}{*}{ Emoticonos/emojis } & \multicolumn{4}{|c|}{ Acuerdo en identificación de significado \% } \\
\hline & Alegría & Enojo & Tristeza & Confuso \\
\hline :-) & 94,8 & 2,6 & 0 & 2,6 \\
\hline$\because$ & 100 & 0 & 0 & 0 \\
\hline (3) & 100 & 0 & 0 & 0 \\
\hline$>:-($ & 0 & 79,5 & 10,3 & 10,2 \\
\hline$\because$ & 0 & 100 & 0 & 0 \\
\hline$\because$ & 0 & 100 & 0 & 0 \\
\hline$:-($ & 0 & 5,1 & 92,3 & 0 \\
\hline (1) & 0 & 2,6 & 97,4 & 0 \\
\hline et) & 0 & 5,1 & 94,9 & 0 \\
\hline
\end{tabular}


La razón de elegir estos elementos se basa en la investigación desarrollada por Rezabeck y Cochenour (1998) que afirmaban que, aunque la plataforma Listservs contenía una variedad mayor de emoticonos, eran estos los emoticonos que aparecían con mayor frecuencia, y por tanto tenían la mayor probabilidad de producir el entendimiento más compartido de los significados comunicados a través de ellos.

En esta prueba piloto participaron 40 sujetos jóvenes voluntarios de una ciudad española con una población aproximada de unos 200.000 habitantes. Fueron escogidos aleatoriamente y su perfil general se resume como así: Edad M = 26,3 (DT = 7,26); 70\% procedencia de cultura española y 30\% culturas asiáticas; $71,8 \%$ estudiantes y $28,2 \%$ trabajadores; $60 \%$ mujeres y $40 \%$ hombres, y con un hábito medio de envío de mensajes online entre 1-3 veces al día por CMC o SNS (Social Networking Service). Los resultados obtenidos (tabla 1) mostraron altos porcentajes en el acuerdo sobre la identificación del significado de los emoticonos con usos más populares, oscilando entre 79,5\%-100\%. Esto indica la existencia de un nivel muy alto de coincidencia entre los sujetos del estudio piloto en reconocer las señales, entender las emociones humanas que se representaban e identificar el significado en su uso comunicativo.

Este estudio piloto confirma la postulación de Rezabeck y Cochenour (1998) de que los emoticonos de uso más frecuente y extendido, tienen mayor probabilidad de producir el entendimiento compartido de los significados comunicados a través de ellos (Tabla 1).

La verificación del acuerdo en la identificación del significado de los emoticonos propuestos en el estudio piloto permitió su empleo posterior en el siguiente diseño instrumental del presente estudio.

\section{Estudio de Encuesta y Diseño Instrumental}

Se diseñó un cuestionario con treinta y cinco afirmaciones situacionales, habituales en la vida cotidiana de cualquier ser humano. El objetivo era conocer las preferencias de su uso por los usuarios al elegir los emoticonos o emojis para acompañar los mensajes textuales que mejor comunicaran su estado de ánimo en el momento de enviar dichos mensajes mediante CMC o SNS. Estas afirmaciones fueron agrupadas como ítems en los siguientes apartados:

\section{-Ítems de mensaje comunicativo de situaciones de la emoción alegre}

En esta parte del cuestionario, se pregunta a los participantes sobre su preferencia en el uso de los emoticonos para expresar y comunicar su sentimiento mediante mensajes online ante doce situaciones en las que ellos probablemente se sentirían contentos, alegres, felices y optimistas. Por ejemplo: «He sido premiado por un trabajo bien hecho» $\mathrm{O}$ «He tenido una agradable sorpresa inesperada» $\mathrm{O}$ «Recibir amor, cariño o muestra de afecto», etc. Para indicar su respuesta a cada ítem de las doce situaciones de alegría, los participantes utilizaron una escala nominal con diferentes emoticonos alegres proporcionados en el cuestionario. Los participantes podían elegir dichos emoticonos/emojis según el orden de su preferencia para expresar y comunicarse a través de mensajes en CMC o SNS: $1=$ «ninguno», es decir, basta con el mensaje en texto verbal solo; $2=:-) ; 3=0 ; 4=0$.

\section{-Ítems de mensaje comunicativo de situaciones de la emoción triste}

Para averiguar el patrón de uso preferente de los emoticonos con los que los participantes expresan y comunican mediante el envío de mensajes en COM o SNS sobre la emoción triste que ellos sienten, se les plantean doce situaciones tristes en las que los seres humanos pueden sentirse afectados, por ejemplo: «La muerte de un ser querido» o «Vivir en soledad» o «Terminar una relación». Los sujetos marcaron según el orden de su preferencia al uso de los emoticonos/emojis presentados en la 
escala para expresar y comunicarse a través del envío de mensajes en CMC o SNS sobre cada ítem de las doce situaciones de tristeza: $1=$ «ninguno» (solo con el mensaje en texto verbal); $2=:-(; 3=: ;$; 4 $=\infty$.

\section{- Ítems de mensaje comunicativo de situaciones de la emoción enojosa}

Consiste en 6 ítems que describen las situaciones ante las que una persona puede experimentar y, probablemente, también reaccionar con ira, rabia o un sentimiento enojoso. Por ejemplo, «Siento impotencia ante las actuaciones de los demás que son incorrectas, ilegales o contrarias al sentido común según mi juicio, pero no puedo hacer nada y tengo que aguantarlas» $\mathrm{O}$ «Me han faltado el respeto». A los participantes se les facilitó una escala de diferentes emoticonos/emojis de los que deberían elegir según el orden de su preferencia para responder cada uno de los seis ítems relacionados al sentimiento de enojo: $1=$ «ninguno» (solo se usa mensaje en texto verbal); $2=$ : $-\left(3={ }^{\circ}\right.$ y $4={ }^{\circ} \odot$.

\section{- Variables hábito de uso TIC}

Se ha incluido en el cuestionario un apartado con tres ítems sobre los hábitos de uso de redes sociales por smartphone, Internet, búsqueda información en páginas web. También se proponía otro ítem sobre el tiempo de permanencia online de los participantes en cada acceso al Internet, además de otro ítem que interrogaba a los participantes sobre su hábito de uso de un idioma extranjero para la comunicación en CMC y SNS.

\section{- Variables demográficas}

Se recogieron las informaciones de los participantes a través de cuatro ítems demográficos: edad, género, procedencia cultural y situación laboral o estudio.

\section{Participantes}

En esta encuesta respondieron al cuestionario arriba descrito un total de 140 sujetos usuarios de Internet por CMC y SNS o SMS por teléfonos móviles. Los participantes fueron voluntarios compuestos por usuarios de comunicación online, estudiantes universitarios de grados y de diferentes programas de máster con edades comprendidas entre 18 y 41 años, una edad M = 23,14 y DT = 4,02. La distribución de género resultó con un $57,1 \%$ femenino y $42,9 \%$ masculino; respecto a la procedencia cultural, era española $=58,3 \%$ y china $=41,8 \%$. Su dedicación profesional se presentaba como: estudio postgrado $=86,3 \%$; grado universitario $=2,1 \%$; trabajo y estudio $=11,7 \%$. Los participantes fueron reclutados en las bibliotecas y aulas universitarias en territorio español.

\section{RESULTADOS}

\section{Análisis Preliminares}

Mediante técnica de tabla de contingencia, se realizaron análisis preliminares obteniendo los resultados descriptivos expuestos a continuación.

\section{- Uso de texto, emoticono o emoji en mensajes de enojo}

En primer lugar, se analizan las frecuencias con las que los encuestados utilizan signos noverbales tales como el código ASC-II por teclado :- $\left(\right.$, el emoticono angry ${ }^{\circ}$ o el emoji enojoso 
para trasmitir mensajes por CMC sobre situaciones cotidianas de enojo que se les planteaban en el cuestionario.

En este análisis, con la excepción del item 3 «Las cosas no marchan tan bien como yo esperaba» donde los usuarios eligen el emoji angry (39,3\%) como preferente, los demás datos obtenidos indican que los encuestados prefieren el uso del estilo comunicativo verbal directo para comunican su estado emocional enojoso en tres de los seis ítems con 45\%, 37,9\% y 42,9\% respectivamente, presentando la significación estadística a nivel entre $p<, 001 \mathrm{y} p<, 01$. En la prueba multivariable, se obtiene el resultado de $\chi^{2}(3)=19,51 ; p<, 001 ;$ Kendall's $W=, 051$ (Tabla 2).

Tabla 2. Uso preferente de texto verbal, signo o emoticono en mensajes enojosos en CMC (\%)

\begin{tabular}{|c|c|c|c|c|c|c|}
\hline Item de mensaje enojoso & $\begin{array}{l}\text { Texto } \\
\text { único }\end{array}$ & r:- ( & $\ddot{i}$ & $\because$ & $\chi^{2}$ & $p$ \\
\hline 1) Me suceden una y otra vez cosas negativas similares. & 32,9 & 18,6 & 26,4 & 22,1 & 6,34 & ,096 \\
\hline 2) Me han faltado el/al respeto. & 45,0 & 12,1 & 15,7 & 27,1 & 36,74 & ,000 \\
\hline 3) Las cosas no marchan tan bien como yo esperaba. & 30,7 & 22,9 & 39,3 & 7,1 & 31,37 & 000 \\
\hline $\begin{array}{l}\text { 4) Algo o alguien me ha interferido o interrumpido el trabajo o } \\
\text { actividad que yo estaba realizando. }\end{array}$ & 29,3 & 17,9 & 31.4 & 21,4 & 6,91 & ,075 \\
\hline $\begin{array}{l}\text { 5) Tengo que soportar el mal estar o dolor físico o someterme bajo } \\
\text { estrés mental. }\end{array}$ & 37,9 & 22,9 & 23,6 & 15,7 & 14,45 & ,002 \\
\hline $\begin{array}{l}\text { 6) Siento impotencia frente a las actuaciones que, según mi juicio, } \\
\text { son incorrectas, ilegales o contrarias al sentido común, pero } \\
\text { debo someterme a ellas. }\end{array}$ & 42,9 & 17,1 & 21,4 & 18,6 & 24,34 & ,000 \\
\hline
\end{tabular}

$$
N=140, \chi^{2}(3)=19,51 ; p<, 001 ; \text { Kendall's W = ,051 }
$$

\section{- Uso de texto, emoticono o emoji en mensajes de alegtía}

Tabla 3. Uso preferente de texto verbal, signo o emoticono en mensajes alegres en CMC (\%)

\begin{tabular}{|c|c|c|c|c|c|c|}
\hline Item mensaje alegre & $\begin{array}{l}\text { Texto } \\
\text { único }\end{array}$ & :-) & U & (30) & $\chi^{2}$ & $p$ \\
\hline 7) He sido premiado por haber realizado un buen trabajo. & 12,1 & 14,3 & 29,3 & 44,3 & 37,54 &, 000 \\
\hline 8) El resultado obtenido ha sido lo que esperaba. & 11,4 & 20,7 & 44,3 & 23,6 & 32,28 & 000 \\
\hline 9) Me elogian con mucho aprecio. & 16,4 & 23,6 & 35,0 & 24,3 & 9,92 & 019 \\
\hline 10) Conseguí lo que siempre había deseado. & 12,1 & 17,6 & 28,6 & 42,1 & 29,88 & 000 \\
\hline 11) He tenido una agradable sorpresa inesperada. & 12,1 & 16,4 & 30,7 & 40,0 & 27,99 & 000 \\
\hline $\begin{array}{l}\text { 12) He experimentado una sensación muy placentera física y } \\
\text { mentalmente. }\end{array}$ & 19,3 & 20,7 & 32,9 & 25,7 & 6,40 & 093 \\
\hline 13) Me han devuelto el favor por lo que les hice. & 27,9 & 27,9 & 32,9 & 10,7 & 15,90 & ,001 \\
\hline 14) Recibir amor, cariño o muestra de afecto. & 12,1 & 17,9 & 32,9 & 37,1 & 23,82 & 000 \\
\hline 15) Reunirme con amigos o familiares. & 29,3 & 10,7 & 37,9 & 22,1 & 22,17 & 000 \\
\hline 16) He recibido unos regalos. & 19,3 & 13,6 & 36,4 & 28,6 & 17,48 & 000 \\
\hline 17) La realidad excede a las expectativas. & 18,6 & 20,7 & 25,0 & 35,0 & 9,0 & 029 \\
\hline 18) He tenido buenas noticias. & 13,6 & 17,1 & 37,9 & 31,4 & 22,34 & 000 \\
\hline
\end{tabular}

En este análisis se observa que los encuestados optan por aplicar el emoticono smiley $\cup$ (entre $32,9 \%-44,3 \%$ ) y el emoji facial alegre (entre 35\%-44,3\%) respectivamente con mayor frecuencia para la comunicación online de su estado de emoción de alegría experimentada en situaciones positivas (Tabla 3).

Los resultados arriba aportan la significación estadística entre nivel $p<, 001$ y $p<, 05$ respectivamente, obteniendo $\chi^{2}(3)=62,61 ; p<, 001 ;$ Kendall's $W=, 11$ en la prueba multivariable. 


\section{- Uso de signos no-verbales en mensajes de tristeza}

En este análisis, se observa que, a diferencia de la emoción alegre en lugar de recurrir a los signos no-verbales, los participantes encuestados prefieren comunicar los mensajes por CMC con texto verbal sobre su sentimiento personal de tristeza en la mayoría de situaciones realistas planteadas en el cuestionario (entre 34,5\%-67,1\%) (Tabla 4). Estos resultados presentan niveles de significación estadística que oscilan entre $p<, 001$ y $p<, 01$. Mediante un análisis de prueba multivariable, estos datos presentan el resultado con $\chi^{2}(3)=62,61 ; p<, 001$; Kendall's $W=, 16$ en los ítems de mensajes tristes.

Tabla 4. Uso preferente de texto verbal, signo o emoticono en mensajes tristes en CMC $(\%)$

\begin{tabular}{|c|c|c|c|c|c|c|}
\hline Ítems mensaje triste & $\begin{array}{l}\text { Texto } \\
\text { único }\end{array}$ & $:-($ & 11 & (6) & $\chi^{2}$ & $p$ \\
\hline 19) Las cosas no han ido bien, el resultado fue decepcionante. & 27,3 & 26,6 & 29,5 & 16,5 & 5,54 & 136 \\
\hline 20) La muerte de un ser querido. & 67,1 & 8,6 & 7,9 & 16,4 & 135,14 &, 000 \\
\hline 21) Terminar una relación, cada cuál por su lado. & 61,4 & 17,9 & 7,9 & 12,9 & 101,88 & 000 \\
\hline 22) Cuando uno se da cuenta de su propia incapacidad. & 42,1 & 23,6 & 26,4 & 7,9 & 33,14 & 000 \\
\hline 23) Oír o ver algo que me da mucha pena y lástima. & 32,9 & 24,3 & 26,4 & 16,4 & 7,71 & 052 \\
\hline 24) Me han reprochado y rechazado. & 43,2 & 19,4 & 25,2 & 12,2 & 19,14 & 000 \\
\hline 25) Sin conseguir lo que uno deseaba. & 34,5 & 21,6 & 32,4 & 11,5 & 18,84 & 000 \\
\hline 26) Las cosas van peor de lo que se esperaba. & 36,2 & 24,6 & 26,1 & 13,0 & 14,92 & 002 \\
\hline $\begin{array}{l}\text { 27) Sentirse mal por haber perjudicado a alguien por los descuidos } \\
\text { cometidos. }\end{array}$ & 38,1 & 19,4 & 24,5 & 18,0 & 14,06 & ,003 \\
\hline 28) Enfermedades graves de las personas cercanas a mí. & 59,5 & 12,1 & 15,0 & 13,6 & 88,0 & 000 \\
\hline 29) La soledad. & 46,8 & 20,9 & 18,0 & 14,0 & 36,28 &, 000 \\
\hline 30) Resultado de una relación de causa-efecto injusta & 43,6 & 23,6 & 20,7 & 12,1 & 29,71 & 000 \\
\hline
\end{tabular}

\section{Estudios Transculturales de Usos de Emoticonos y Textos en Comunicación Online}

En los siguientes estudios transculturales, se ha aplicado la técnica de análisis de varianza (ANOVA) con el propósito de observar la manera en que la variable cultural podría afectar las expresiones emotivas entre los internautas españoles y chinos en el envío de mensajes online por CMC.

\section{- Prueba de homogeneidad interna de los ítems por aproximación}

Antes de proceder con los estudios de ANOVA, se realizó la técnica de clasificación de dimensión mediante un análisis de cluster (conglomerado) con el fin de reducir los datos. Se comprobó que los ítems de las tres emociones primarias fuesen agrupados por su aproximación. Es decir, los ítems dentro de cada uno de los conglomerados resultaron homogéneos y fueron los más discrepantes de los contenidos del resto (Vilà-Buenos, et al., 2014). De este modo, se obtienen los resultados de los conglomerados que apoyan la agrupación por dimensiones de las tres emociones básicas universales -alegría, enojo, tristeza. El dendrograma resultado de SPSS (Figura 1) muestra que los ítems se agrupan en tres dimensiones de emoción:

\section{Cluster 1: Enojo}

3) Las cosas no marchan tan bien como yo esperaba.

4) Algo o alguien me ha interferido o interrumpido el trabajo o actividad que yo estaba realizando.

1) Me suceden una y otra vez cosas negativas similares. 
5) Tengo que soportar el malestar o dolor físico o someterme bajo estrés mental.

6) Siento impotencia frente a las actuaciones que, según mi juicio, son incorrectas, ilegales o contrarias al sentido común, pero debo someterme a ellas.

2) Me han faltado al respeto.

Figura 1. Análisis Conglomerado

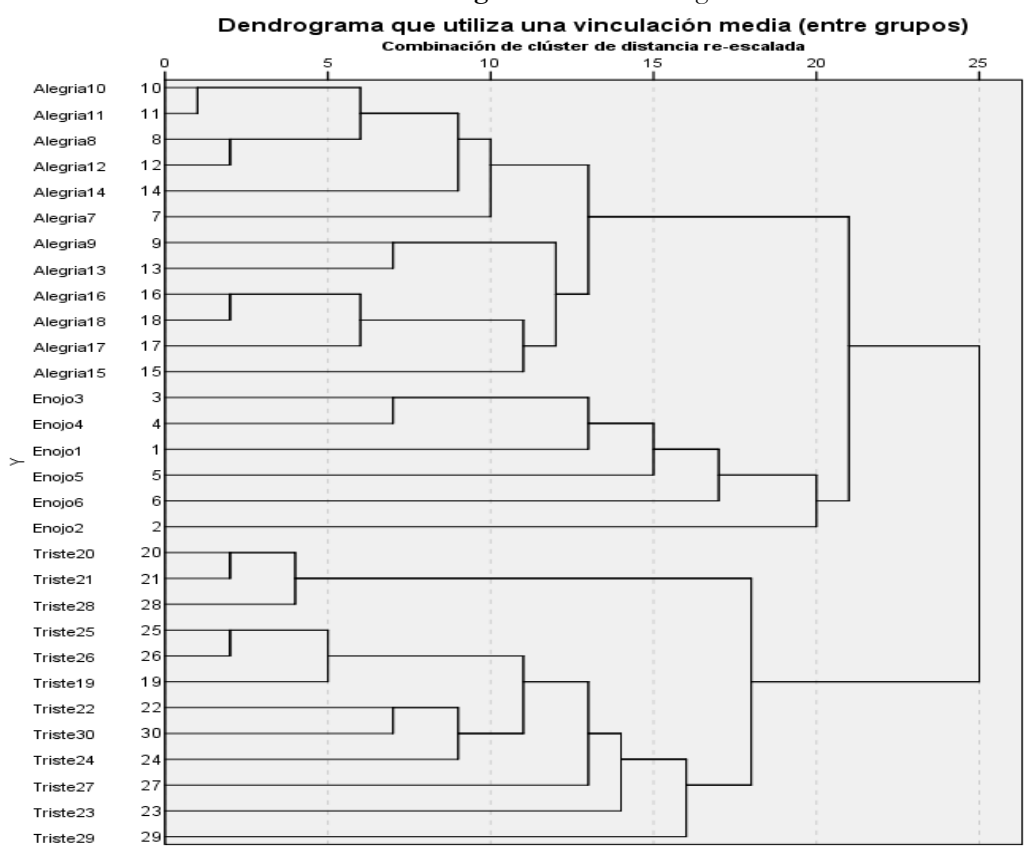

\section{Cluster 2: Alegría}

10) Conseguí lo que siempre había deseado.

11) He tenido una agradable sorpresa inesperada.

8) El resultado obtenido ha sido lo que esperaba.

12) He experimentado una sensación muy placentera física y mentalmente.

14) Recibir amor, cariño o muestra de afecto.

7) He sido premiado por haber realizado un buen trabajo.

9) Me elogian con mucho aprecio.

13) Me han devuelto el favor por lo que les hice.

16) He recibido unos regalos.

18) He tenido buenas noticias.

17) La realidad excede a las expectativas.

15) Reunirme con amigos o familiares.

\section{Cluster 3: Tristeza}

20) La muerte de un ser querido.

21) Terminar una relación, cada cuál por su lado.

28) Enfermedades graves de las personas cercanas a mí.

25) Sin conseguir lo que uno deseaba.

26) Las cosas van peor de lo que se esperaba. 
19) Las cosas no han ido bien y el resultado fue decepcionante.

22) Cuando uno se da cuenta de su propia incapacidad.

30) Resultado de una relación de causa-efecto injusta.

24) Me han reprochado y rechazado.

27) Sentirse mal por haber perjudicado a alguien por los descuidos cometidos.

23) Oír o ver algo que me da mucha pena y lástima.

29) La soledad.

\section{ANOVA: Estudio transcultural del uso de textos, emoticonos y emojis entre españoles vs. chinos en los mensajes emotivos en CMC}

Para desarrollar este estudio, se llevó a cabo un proceso de transformación donde se recodificaron y recalcularon los datos mediante el establecimiento de escalas basadas en medidas tipificadas.

Los estudios de análisis de varianza fueron desarrollados utilizando la cultura «española-china» como variable independiente, $\mathrm{y}$, como variables dependientes, las dimensiones emocionales el «enojo», la «alegría» y «tristeza», con cuatro categorías: «textual»; «emoticono por teclado basado en signo ASCII», «emoticono» $\mathrm{y}$ «emoji facial».

\section{Dimensión Enojo}

Los resultados obtenidos muestran que los usuarios en general prefieren el «texto verbal único» $(M=, 36 ; D T=, 31)$ más que el emoticono basado en «signo ASC-II» $(M=, 19 ; D T=, 20)$, el emoticono «angry» $(M=, 27 ; D T=, 22)$ o «emoji facial enojo» $(M=, 19 ; D T=, 20)$ para comunicar mensajes que implican estados de emoción enojoso que les afectan. No obstante, al comparar las preferencias de los grupos culturales, se observa que los chinos $(m=, 24 ; d t=, 22)$ prefieren el signo ASC-II $:$ :- $($ con mayor medida que los españoles $(m=, 15 ; d t=, 18)$, esta diferencia aporta un nivel de significación estadística a $p<, 01\left(F[1,137]=8,004 ; \eta^{2}=, 06\right)$. Por otra parte, los españoles $(m=, 24 ; d t=, 22)$ optan por «emoji facial expresivo $\because$ » más que los chinos $(m=, 12 ; d t=, 16)$, con la prueba de significación a $p<, 001\left(F[1,137]=11,961 ; \eta^{2}=, 08\right)($ Tabla $5-1)$.

\section{Dimensión Alegría}

El conjunto de los usuarios expresa como primera preferencia el uso del «emoticono smiley $\Theta »(M$ $=$,34; $D T=$,22) para mostrar su alegría en los mensajes comunicativos online por delante del uso de «texto verbal único» $(M=, 17 ; D T=, 21)$, el signo ASC-II $(M=, 18 ; \mathrm{DT}=, 20)$ o «emoji facial alegre » $(M=, 30 ; D T=, 24)$. En esa dimensión «alegría» se observa que los españoles $(m=, 20 ; d t=, 23)$ tienden a utilizar más «texto verbal único» que los chinos $(m=, 13 ; d t=, 18)$ con una significación marginal a $p<0,1$ (Tabla 5-2).

\section{Dimensión Tristeza}

En situaciones de tristeza, los usuarios encuestados en general muestran su preferencia por utilizar únicamente el texto verbal $(M=, 44 ; D T=, 29)$ para su comunicación online. Se halla que los españoles optan por transmitir su tristeza vía CMC mediante textos verbales únicamente $(m=, 53$; $\mathrm{dt}=$ ,27) más que los chinos $(m=, 32 ; d t=, 27)$. La diferencia se muestra significativa a $p<, 001(F[1,137]=$ 20,$\left.185 ; \eta^{2}=, 13\right)$. Por otro lado, los chinos utilizan más el emoticono con los signos ASC $(m=, 27 ; d t$ $=, 19)$ más que los españoles $(\mathrm{m}=, 15 ; d t=, 17)$ de forma significativa $\left(p<, 001 ; F[1,137]=13,952 ; \eta^{2}\right.$ $=, 09)$. 
Tabla 5. Resultados de ANOVA del uso preferente de textos verbales, signos no-verbales, emoticonos o emojis entre usuarios españoles y chinos para expresar las emociones en CMC

\begin{tabular}{|c|c|c|c|c|c|c|c|c|c|}
\hline \multirow[t]{2}{*}{ 1. Dimensión Enojo } & \multicolumn{2}{|c|}{ Total } & \multicolumn{2}{|c|}{ Españoles } & \multicolumn{2}{|c|}{ Chinos } & \multirow{2}{*}{$\begin{array}{c}F \\
(1,137) \\
\end{array}$} & \multirow[b]{2}{*}{$p$} & \multirow[b]{2}{*}{$\eta^{2}$} \\
\hline & $M$ & $D T$ & $m$ & $d t$ & $m$ & $d t$ & & & \\
\hline Solo texto verbal enojoso & ,36 & ,31 &, 38 &, 30 & ,34 & ,32 & ,498 & ,481 & ,004 \\
\hline$>:-($ & ,19 & ,20 & ,15 & ,18 & ,24 & ,22 & 8,004 & ,005 &, 06 \\
\hline &, 27 & ,22 &, 24 & ,20 &, 30 &, 24 & 2,390 & ,124 & ,02 \\
\hline$\because$ & ,19 & ,20 & ,24 & ,22 & ,12 & ,16 & 11,961 & ,001 & 08 \\
\hline \multicolumn{10}{|l|}{ 2. Dimensión Alegría } \\
\hline Solo texto verbal alegre & ,17 &, 21 & ,20 & ,23 & ,13 & ,18 & 2,877 & ,092 & ,021 \\
\hline$\left.:^{-}\right)$ & ,18 & ,20 &, 17 & ,22 & ,20 & ,17 & 687 & ,409 &, 005 \\
\hline & ,34 & ,22 &, 32 &, 22 & ,36 & ,21 & 1,330 & ,251 & 01 \\
\hline &, 30 & 24 &, 31 & ,24 &, 29 &, 24 & ,188 & ,665 & ,001 \\
\hline \multicolumn{10}{|l|}{ 3. Dimensión Tristeza } \\
\hline Solo texto verbal triste & ,44 &, 29 &, 53 &, 27 &, 32 &, 27 & 20,185 &, 000 &, 13 \\
\hline$:-($ & ,20 & ,19 & ,15 &, 17 & ,27 & ,19 & 13,952 &, 000 & 09 \\
\hline & ,22 & ,17 &, 19 & ,17 & ,26 & ,18 & 5,589 & ,019 &, 04 \\
\hline (c) & ,14 &, 17 &, 13 & ,17 & ,15 & ,18 &, 504 & ,479 & ,004 \\
\hline
\end{tabular}

$\mathrm{Al}$ mismo tiempo, los chinos también muestran que prefieren el uso de «emoticono frowny : ${ }_{\gg}(m$ $=26 ; d t=, 18)$ más que los españoles $(m=, 19 ; d t=, 17)$ de manera significativa $(p<, 05 ; F[1,137]=$ 5,$\left.589 ; \eta^{2}=, 09\right)$. No obstante, la diferencia del uso de «emoji facial triste ${ }^{\bullet} \gg$ entre los chinos $(m=, 15$; $d t=, 18)$ y los españoles $(m=, 13 ; d t=, 17)$ no resultó significativa (Tabla 5-3).

\section{DISCUSIÓN}

Los resultados obtenidos tras el análisis vienen a responder al planteamiento investigador previo del presente estudio. Se hace evidente que, a pesar de las crisis y guerras en el mundo, la humanidad desea poder emitir mensajes de esperanza para mantener un ánimo optimista. Se responde a la Pregunta-1 con el resultado de los análisis presentados en la tabla 2 que muestran cómo los usuarios de CMC encuestados en este estudio envían, en general, los 12 tipos de mensajes sobre situaciones positivas en las que se encuentran. La mayoría de las veces, no solo mandan mensajes verbales positivos, sino que también los refuerzan acompañando con signos no-verbales como el emoticono smiley o emoji facial alegre. Una simple carita feliz puede hacer más amigable un mensaje y darle un tono más amable. Estos resultados son congruentes con otros estudios anteriores que han demostrado que el uso de los emoticonos tiene un alto poder comunicativo en las redes sociales, porque pueden capturar la atención más rápido que un simple texto y contagiar ampliamente los sentimientos positivos.

En comparación con la comunicación de mensajes positivos, la Pregunta-2 plantea si los usuarios en general reducen la frecuencia del uso de los signos tristes no-verbales para comunicar mensajes tristes por CMC a fin de evitar que se enfatice el sentimiento de tristeza en dichos mensajes. Efectivamente, en el presente estudio se observa que los usuarios CMC reducen en general y en gran medida el uso de los signos no-verbales, el de los emoticonos basados en ASC-II por teclado $(\mathrm{M}=, 20)$; el emoticono frowny $(M=, 22)$ y el emoji facial triste $(M=, 14)$ para comunicar mensajes tristes. En estas circunstancias prefieren, en cambio, expresarse con textos verbales directos $(M=, 44)$. 
Igualmente se confirma en nuestro estudio el planteamiento de la Pregunta-3. Los resultados del análisis muestran que los usuarios de CMC optan en general por emitir mensajes de enojo mediante texto verbal $(\mathrm{M}=, 36)$ y directamente expresados, por encima del recurso a los signos no-verbales tales como el emoticono con signos en teclado ASC-II $(\mathrm{M}=, 19)$; el emoticono angry $(\mathrm{M}=, 26)$ y emoji facial enojosa $(M=, 18)$.

Asimismo, la Pregunta-4 queda respondida con los datos que muestran que los usuarios en general y sin distinción, prefieren el uso de textos verbales $(\mathrm{M}=, 36)$ para comunicar los mensajes enojoso. No obstante, al comparar los signos no-verbales más usados por los dos grupos de participantes, se observa que los chinos prefieren el signo ASC-II s:- ( en mayor medida $(m=, 24)$ que los españoles $(m$ $=, 15)$. En cambio, los españoles optan por «emoji facial enojoso $\because »(m=, 24)$ más que los chinos $(m$ $=, 12$ ). Estos datos son significativos estadísticamente a nivel de $p<, 01 \mathrm{y} \mathrm{p}<, 001$ respectivamente.

En cuanto al planteamiento en la Pregunta-5 sobre si el bagaje cultural puede influir en los usuarios de CMC al transmitir mensajes online sobre la emoción de alegría, los resultados del análisis indican que los dos grupos culturales de participantes prefieren indistintamente el emoticono smiley y emoji facial alegre expresivo. Esto confirma la universalidad en la comunicación online de los buenos sentimientos sobre situaciones positivas.

Por ultimo, nuestros hallazgos pueden aclarar la cuestión cultural planteada en la pregunta-6 sobre la diferencia entre los españoles y chinos. Los resultados señalan en general que los dos grupos utilizan los textos de modo preferente, aunque los españoles muestran el uso del «texto verbal único» ( $\mathrm{m}=$ ,53) en una significativa mayor medida que los chinos $(m=, 32)$. Sin embargo, respecto a todos los usos no-verbales de los emoticonos de «signo ASC-II :-( por teclado», y «frowny •̈» o «emoji facial triste $\bullet \gg$ para la comunicación online de mensajes de la tristeza, los chinos los utilizan en mayor medida que los españoles.

En resumen, los resultados de este estudio, fundamentado sobre supuestos de estudios anteriores, vienen a verificar que, a diferencia de la universalidad en la atribución de la emoción alegre a situaciones positivas vividas por los individuos, es evidente la existencia de diferencias culturales para la comunicación de la tristeza. En este aspecto, el estilo comunicativo observado entre los encuestados españoles se presenta algo más posicionado hacia el polo [Bajo Contexto] cultural -sobre la escala continua de la variabilidad cultural- en comparación con el estilo comunicativo de la cultura china, cuyo prototipo cultural se posiciona en el polo opuesto (Alto Contexto] en el que destaca el estilo indirecto de comunicación.

La observación anterior es especialmente evidente en el presente estudio cuando se aborda la comunicación de tristeza a través del uso de los textos verbales $v$ s. los signos no-verbales en los mensajes por CMC. Puesto que el estilo comunicativo de alto contexto en cultura colectivista (Hall, 1976) aporta una mayor información relacionada con el contexto físico, son los individuos de culturas de alto contexto, (AC) como los sujetos chinos, los que depositan mayor confianza en la comunicación noverbal que en la comunicación verbal, mientras que el estilo comunicativo de bajo contexto (BC) característico de culturas individualistas es identificado de forma contraria. Es decir, el significado de los mensajes se interpreta mediante el contenido de lo escrito o de que se ha dicho por la comunicación verbal.

Lo mismo ocurre con la comunicación de cara a cara: las culturas AC tienden a usar estrategias no-verbales para transmitir el significado que podrían aportar los gestos, el lenguaje corporal, el silencio o actuaciones simbólicas. En el presente estudio, se ha podido comprobar que los sujetos de cultura china, más colectivista, recurren a usar los emoticonos en general, y no tanto los emojis faciales como se esperaba, con mayor frecuencia e intensidad que los españoles también en el intercambio de 
mensajes mediante CMC y SNS. En cambio, entre los usuarios con tendencia cultural relativamente más individualista, como es la española en comparación con la china, su estilo comunicativo también se inclinaría al tipo de bajo contexto (BC).

Finalmente, con respecto al mayor uso del emoji facial enojoso por parte de los encuestados españoles, cabría interpretar que la expresión extrovertida de dicho emojis quedaría en el polo del estilo comunicativo directo, que caracteriza la cultura BC, con la intención de intensificar el sentimiento enojoso de forma más directa e inequívoca en lugar de la insinuación tenue de lo que puedan trasmitir otros emoticonos de símbolos iconográficos.

\section{CONCLUSIÓN}

Este estudio nos ha aportado datos de algunos aspectos del uso de los signos no-verbales, como los «emoticonos» $\mathrm{y}$ «emojis» de los usuarios de CMC y SNS para comunicar mensajes sobre los estados de diferentes emociones primarias universales: alegría, tristeza y enojo. La teoría de la variabilidad cultural incluida en el presente estudio como marco teórico ha servido de base para indagar si existen modelos distintos de usos preferentes de estos signos no-verbales vs. la opción del uso de texto verbal único entre los usuarios de diferencias procedencias culturales durante el proceso de interacción mediante los mensajes para la comunicación online, CMC y/o SNS, de las situaciones de emoción que sienten.

Se ha aplicado la técnica de análisis de conglomerado (cluster analysis) en el proceso metodológico. Con ello se realizó la comprobación de consistencia interna de los ítems con escala de medida nominal del cuestionario. Gracias a esta técnica, dichos ítems se agrupan efectivamente por su aproximación entre sí para establecer tres dimensiones de emoción, alegría, tristeza y enojo, que son consideradas por los psicólogos sociales las tres emociones primarias humanas, y compartidas por todas las personas, independientemente de su precedencia cultural. A diferencia de estas emociones primarias, universalmente compartidas, existen otras como la de culpa, asco, vergüenza, etc. que los expertos sostienen que son culturalmente específicas y, por tanto, deben de ser estudiadas en determinados contextos culturales.

La confirmación del establecimiento de las tres diferentes dimensiones, claramente definidas, ha permitido a la analista investigadora del presente estudio a proceder mediante los estudios de ANOVA para examinar la existencia o no de patrones culturales en el uso de los signos no-verbales, en forma de emoticonos, entre los usuarios con diferente procedencia cultural, para comunicar e intercambiar mensajes personales relacionados a las emociones.

Los hallazgos distan de ser considerados como definitivos debido, por un lado, al modesto número de participantes $(\mathrm{N}=140)$ que colaboraron respondiendo al cuestionario durante el desarrollo de la encuesta, y, por el otro, a la gama necesariamente reducida de los emoticonos y emojis considerados en el diseño instrumental de la investigación. Además, la utilización del castellano en lugar de la lengua china podría causar alguna variación en la interpretación del significado exacto de los ítems por parte de los participantes chinos, a pesar de ser estudiantes en activo en universidades españolas. Limitaciones aparte, los datos obtenidos de los análisis nos orientan en el conocimiento de qué contexto emocional prefieren usar los usuarios de CMC y/o SNS de diferente cultura y qué tipo de emoticono/ emoji expresivo vs. texto único priorizan en los mensajes online para comunicar sus estados de emoción. 


\section{BIBLIOGRAFÍA}

Adrianson, L. (2001). Gender and computer-mediated communication: Group processes in problem, Computers in Human Behavior, 17(1), 71-94.

Amaghlobeli, N. (2012). Linguistic features of typographic emoticons in SMS discourse. Theory and Practice in Language Studies, 2(2), 348-354.

Constantin, C., Kalyanaraman, S., Stavrositu, C. y Wagoner, N. (2002). To be or not to be emotional: Impression formation effects of emoticons in moderated chatrooms. Paper presented at the Communication Technology and Policy Division at the 85th Annual Convention of the Association for Education in Journalism and Mass Communication, Miami Beach, Florida, in 2002 August (Fecha de consulta, 08/11/2015).

Crystle, D. (2001). Language and the Internet. Cambridge: Cambridge University Press.

Derks, D., Fischer, A. H. y Bos A. E. R. (2007). The role of emotion in computer-mediated communication: A review. Computers in Human Behavior, doi: https://doi.org/10.1016/i.chb.2007.04.004. (Fecha de consulta, 09/12/2015).

Dresner, E. y Herring, S. C. (2010). Functions of the nonverbal in CMC: Emoticons and illocutionary Force. Communication Theory, 20, 249-268. doi: https://doi.org/10.1111/j.1468-2885.2010.01362.x

Duggan, B. U. M. y Smith, A. (2013). Social media update 2013. Pew Research Internet Project, 7-9.

Ekman, P. (1993). Facial expression and emotion. American Psychologist, 48(4), 384-392.

Ekman, P. y Oster H. (1997). Expresiones faciales de la emoción. Annual Review of Psychology, 30, $527-$ 554.

Ellensburg, R. (2012). Emoticons as social information: The effect of emoticons on interpersonal perception in computer-mediated business communication. TFM de programa de investigación en psicología, Universidad de Amsterdam. Disponible en:

http://dare.uva.nl/cgi/arno/show.cgi?fid=463478 (Fecha de consulta 06/02/2016).

Fahlman, S. E. (1982). Smiley Lore :-) (inédito). https://www.cs.cmu.edu/ sef/sefSmiley.htm (Fecha de recuperación, 10/06/2015).

Fernández, I., Zubieta, E. y Páez, D. (2000). Expresión e inhibición emocional en diferentes culturas. En D. Páez y M. M. Casullo (Comps.), Cultura y alexitimia: ¿Cómo expresamos aquello que sentimos? (pp. 73-98). Buenos Aires: Paidós.

Ferris, S. (1996). Women on-line: Cultural and relational aspects of women's communication in online discussion. Interpersonal Computing and Technology, 4(3-4), 29-40. Disponible en: http://www.helsinki.fi/science/optek/1996/n3/ferris.txt (Fecha de consulta, 08/08/2015).

Gudykunst, W. B. (1997). Cultural variability in communication: An introduction. Communication Research, 2(4), 327-348.

Gudykunst, W. B., Lee, C. M., Nishida, T. \& Ogawa, N. (2005). Theorizing about intercutural communication. Thousand Oaks, CA: Sage.

Gudykunst, W. B., Matsumoto, Y., Ting-Toomey, S., Nishida, T., Kim, K. y Heyman, S. (1996). The influence of cultural individualism-collectivism, self construals, and individual values on communication styles across cultures. Human Communication Research, 22(4), 510-543.

Hall, E. T. (1976). Beyond culture. Garden City, NY: Doubleday.

Hall, E. T. y Hall, M. R. (1990). Understanding cultural differences. Germans French and Americans, 54. doi: http://doi.org/10.5860/CHOICE.28-2221.

Hofstede, G. \& Bond, M. H. (1984). Hofstede's culture dimensions. Journal of Cross-Cultural Psychology, 15(4), 417-433. 
Hofstede, G., Hofstede, G. J. y Minkov, M. (2010). Culture and organizations: Soft ware of the mind. International cooperation and its importance for survival. NY: McGraw-Hill Companies.

Huffaker, D. A. \& Calvert, S. L. (2006). Gender, identity and language use in teenage blogs. Journal of Computer Mediated Communication, 10(2). doi: https://doi.org/10.1111/j.1083-6101.2005.tb00238.x

Jenson, S. (2005). Default thinking: Why consumer products fail. En R. Harper, A. Taylor, y L. Palen (Eds.), The inside text: Social perspectives on SMS in the mobile age (pp. 305-325). London: Kluwer.

Jibril, T. A. y Abdullah, M. H. (2013). Relevance of emoticons in computer mediated communication contexts: An overview. Asian Social Science, 9(4), 201-207.

Kasensniemi, E. L. y Rautiainen, P. (2002). Mobile culture of children and teenagers in Finland. N. Y.: Cambridge University Press.

Lee, C. (2003). How does instant messaging affect interaction between the genders? Stanford, CA: The Mercury Project for Instant Messaging Studies at Stanford University. Disponible en:

http://www.stanford.edu/class/pwr3-25/group2/projects/lee.html (Fecha consulta, $08 / 12 / 2015)$.

Ling, R. (2005). Mobile communication vis-à-vis teen emancipation, peer group integration and deviance. En R. Harper, A. Taylor y L. Palen (Eds.), The inside text: Social perspectives on SMS in the mobile age (pp. 175-189). London: Kluwer.

Lo, S. (2008). The nonverbal communication functions of emoticons in computer-mediated communication. Cyber Psychology \& Behavior, 11(5), 595-597.

Páez, D., Fernández, I., Ubillos, S. y Zubieta, E. (Eds.). (2004). Psicología social, cultura y educación. Madrid: Pearson Educación.

Provine, R. R., Spencer, R. J. y Mandell, D. L. (2007). Emotional expression online: Emoticons punctuate website text messages. Journal of Language and Social Psychology, 26, 299-307.

Ptaszynski, M., MacIejewski, J., Dybala, P., Rzepka, R. y Araki, K. (2010). CAO: A fully automatic emoticon analysis system based on theory of kinesics. IEEE Transactions on Affective Computing, 1(1), 46-59.

Qiao, Q. (2010). The adoption and use of SMS among Chinese teenagers. Memoire: Universite de Montreal. Disponible en: https://papyrus.bib.umontreal.ca/xmlui/handle/1866/3554 (Fecha consulta, 06/02/2016).

Rezabek, L. L. y Cochenour, J. J. (1998). Visual cues in computer-mediated communication: Supplementing text with emoticons. Journal of Visual Literacy, 18, 201-215.

Thompson, P. A. \& Foulger, D. A. (1996). Effects of pictographs and quoting on flaming in electronic mail. Computer in Human Behavior, 12, 225-243.

Tossell, Ch. C., Kortum, P., Shepherd, C., Barg-Walkow, L. H., Rahmati, A. y Zhong, L. (2012). A longitudinal study of emoticon use in text messaging from smartphones. Computer in Human Behavior, 28, 659-663.

Vilà-Baños, R., Rubio-Hurtado, M. J., Berlanga-Silvente, V. y Torrado-Fonseca, M. (2014). Cómo aplicar un cluster jerárquico en SPSS. REIRR, 7(1), 113-127.

Walter, J. B. \& D'Addario, K. P. (2001). The impacts of emoticons on message interpretation in computer-mediated communication. Social Science Computer Review. doi:

http://doi.org/10.1177/089443930101900307 